\title{
213 AB-X INTEGRATED CIRCUIT T CELLS DEMONSTRATE IMPROVED POTENCY, EXPANSION, AND SPECIFICITY COMPARED TO MSLN CAR T CELLS
}

Stephen Santoro*, Aaron Cooper, Natalie Bezman, Jun Feng, Kanika Chawla, Jaspar Williams, John Gagnon, Jason Hall, Dina Polyak, Angela Boroughs, Michelle Nguyen, Suchismita Mohanty, Adam Litterman, Jeff Granja, David DeTomaso, Grace Zheng, Jenessa Smith, Drake LeFace, Tarjei Mikkelsen, Susie Jun. Arsenal Biosciences, South San Francisco, CA, USA

Background In solid tumors, CAR T cell efficacy is limited by off-tumor toxicity and suppression by the tumor microenvironment (TME). $\mathrm{AB}-\mathrm{X}$ is an integrated circuit $\mathrm{T}$ cell (ICT cell) intended for the treatment of ovarian cancer. AB-X includes a transgene cassette with two functional modules: 1) an "AND" logic gate designed to limit off-tumor toxicity through dual tumor antigen recognition; 2) a dual shRNA-miR to resist TME suppression and improve ICT cell function. The AB-X logic gate consists of a priming receptor that induces expression of an anti-mesothelin (MSLN) CAR upon engagement of a ALPG/P (alkaline phosphatase germ-line/placental). The dual shRNA-miR mediates downregulation of FAS and PTPN2. The $\mathrm{AB}-\mathrm{X}$ DNA cassette is inserted into the $\mathrm{T}$ cell genome at a defined novel genomic site via CRISPR-based gene editing.

Methods Dual-antigen specificity of the logic gate was assessed in mice harboring MSLN+ and ALPG/P+MSLN+ K562 tumors established on contralateral flanks. Potency was measured in a subcutaneous MSTO xenograft model. Logic-gated ICT cells were compared with MSLN CAR T cells in both models. In vitro, expansion of ICT cells with the FAS/PTPN2 shRNA-miR was evaluated in a 14 day repetitive stimulation assay (RSA). In vivo, expansion and potency were measured in the MSTO xenograft model. An in vitro FAS cross-linking assay was conducted to assess the impact of FAS knockdown on FAS-mediated apoptosis.

Results Logic-gated ICT cells demonstrated specific activity against ALPG/P+MSLN+ tumors, but had no effect against MSLN+ tumors in the K562 in vivo specificity model. In addition, logic-gated ICT cells demonstrated greater in vivo potency than MSLN CAR T cells in the MSTO xenograft model. In our RSA, ICT cells containing the FAS/PTPN2 shRNA-miR had 8-fold greater expansion than the MSLN CAR T cells. Enhanced expansion was confirmed in vivo with ICT cells demonstrating $>10$-fold expansion in tumors and peripheral blood, enabling comparable growth inhibition in MSTO xenografts at less than one quarter the dose of the MSLN CAR T cells. Importantly, PTPN2 knockdown resulted in balanced expansion of all $\mathrm{T}$ cell subsets, including CD45RA +, CCR7+ memory cells. Lastly, ICT cells containing the FAS/PTPN2 shRNA-miR were resistant to FAS-mediated apoptosis.

Conclusions AB-X ICT cells specifically recognize ALPG/P +MSLN+ tumors, demonstrate superior potency, expansion, and persistence compared with MSLN CAR T cells, and are resistant to ovarian TME suppression. AB-X will be evaluated in clinical trials for treatment of platinum resistant/refractory ovarian cancer.

Acknowledgements We would like to acknowledge all of our colleagues at Arsenal Biosciences, without whom this work would not have been possible.

http://dx.doi.org/10.1136/jitc-2021-SITC2021.213 